\title{
Le territoire, générateur d'inégalités face aux cancers
}

Yohan Fayet, Virginie Chasles, Françoise Ducimetière et Pr Isabelle RayCoquard

\section{OpenEdition}

Journals

Édition électronique

URL : http://journals.openedition.org/rfst/700

DOI : $10.4000 /$ rfst. 700

ISSN : 2492-3672

Éditeur

Espaces et SOciétés (UMR 6590)

\section{Référence électronique}

Yohan Fayet, Virginie Chasles, Françoise Ducimetière et Pr Isabelle Ray-Coquard, « Le territoire, générateur d'inégalités face aux cancers », Revue francophone sur la santé et les territoires [En ligne], Miscellanées, mis en ligne le 17 janvier 2018, consulté le 06 avril 2021. URL : http:/l journals.openedition.org/rfst/700; DOI : https://doi.org/10.4000/rfst.700

Ce document a été généré automatiquement le 6 avril 2021.

\section{(i) (2)}

La Revue francophone sur la santé et les territoires est mise à disposition selon les termes de la Licence Creative Commons Attribution - Pas d'Utilisation Commerciale - Partage dans les Mêmes Conditions 4.0 International. 


\title{
Le territoire, générateur d'inégalités face aux cancers
}

\author{
Yohan Fayet, Virginie Chasles, Françoise Ducimetière et Pr Isabelle Ray- \\ Coquard
}

\section{Introduction}

1 De par leur importance et leur progression, les inégalités face aux cancers constituent un enjeu majeur de santé publique depuis plusieurs années. Elles participent, en effet, à la persistance des inégalités de santé dans les pays développés (Mackenbach, 2012) et notamment en France où ces inégalités sont parmi les plus élevées d'Europe (Leclerc, Kaminski, \& Lang, 2008). La dimension géographique de ces inégalités est aujourd'hui assez bien documentée, grâce aux multiples cartographies utilisant des données d'incidence (Rican, Salem, Roudier-Daval, \& Weill, 2004) et de mortalité (Salem, Rican, Jougla, Suss, \& Berthod-Wurmser, 2000), permettant de localiser les régions françaises les plus durement touchées. L'essor des études de contexte en épidémiologie a aussi permis d'identifier les caractéristiques géographiques ayant un impact sur la santé et pouvant donc contribuer aux inégalités face à la maladie. Ces différentes contributions cartographiques et épidémiologiques favorisent la reconnaissance de la territorialité des inégalités face aux cancers. Il semble cependant nécessaire, afin de lutter plus efficacement contre les inégalités territoriales face aux cancers, de comprendre davantage comment elles se construisent, en reconstituant plus précisément le processus aboutissant à ces inégalités. Cette modélisation nécessite, d'une part, une approche globale et multidimensionnelle du territoire, permettant de produire un découpage géographique pertinent pour l'étude des inégalités de santé.

Elle demande, par ailleurs, de reconstituer, grâce aux données cliniques, l'évolution des chances de survie au cours du parcours médical des patients. La cohorte EMS, regroupant l'ensemble des patients diagnostiqués pour un sarcome (cancer rare) en Rhône-Alpes, a été analysée afin de montrer les perspectives scientifiques offertes par cette approche méthodologique interdisciplinaire. 


\section{Pour une approche globale des effets du territoire sur la santé}

3 Depuis le début des années 2000, le développement important des études contextuelles a confirmé l'intérêt des épidémiologistes pour l'analyse géographique des faits de santé (Riva, Gauvin, \& Barnett, 2007). S'ils ont longtemps été freinés par le biais de «l'erreur écologique ", de multiples études multi-niveaux, distinguant l'influence des caractéristiques individuelles du contexte de vie, ont apporté la preuve statistique d'une territorialité des faits de santé (Ellen, Mijanovich, \& Dillman, 2001 ; Meijer, Röhl, Bloomfield, \& Grittner, 2012). Initiées afin d'améliorer la précision des modèles épidémiologiques en évaluant l'effet de facteurs exogènes connus sur la santé, les études contextuelles ont aussi permis d'explorer et de révéler la diversité des interactions entre territoire et santé (Diez Roux \& Mair, 2010). Grâce à la multiplicité des indicateurs employés et des interactions identifiées dans la littérature, on peut aujourd'hui établir que les caractéristiques physiques, sociales et médicales du territoire participent de concert à la production des inégalités territoriales de santé.

\section{Multiplicité et diversité des impacts du territoire sur la santé}

4 La première dimension par laquelle le territoire génère des inégalités de santé est donc physique car celui-ci, en tant que milieu aménagé, vecteur d'une certaine qualité de vie mais aussi de risques, peut avoir différents effets sur la santé de sa population. En effet, par sa capacité à protéger ses habitants des nuisances et à lui donner accès à certaines aménités, le territoire peut avoir un impact non négligeable, en tant qu'environnement de vie, sur les conditions de vie et la santé de ses habitants. L'influence de ces caractéristiques physiques sur la santé peut être à la fois directe, comme l'exposition aux nuisances (Kihal-Talantikite et al., 2013) ou encore la qualité du logement (Gibson et al., 2011), mais aussi indirecte, en agissant sur les conditions de vie et la perception même du territoire par ses habitants (Maass, Lindstrøm, \& Lillefjell, 2014). Les indicateurs géographiques mesurant ces caractéristiques varient fortement en fonction de la pathologie étudiée et au gré des facteurs de risques préalablement identifiés. Dans le cas du cancer par exemple, l'approche géographique va notamment servir à évaluer le rôle des expositions à des agents chimiques sur l'incidence des cancers, que ce soient les particules issues de la pollution atmosphérique (Künzli et al., 2000), les pesticides (Cabidoche et al., 2009; Saunders et al., 2014). Pour ce qui est des maladies cardiovasculaires et des déterminants du surpoids, les recherches portent par exemple sur la capacité des territoires à favoriser la mobilité (Cervero \& Kockelman, 1997; Leal \& Chaix, 2011 ; Smith et al., 2008) ou encore l'accessibilité des commerces alimentaires (Chaix et al., 2012 ; Holsten, 2009).

5 Au-delà de cette matérialité physique, le territoire est défini aussi comme un espace habité et approprié par une population. Cette dimension sociale du territoire fait que les caractéristiques et les comportements plus ou moins spécifiques de sa population l'exposent à certains risques de santé. De la même façon que Salem montrait la territorialité de certains comportements de santé (Salem, Rican, Kürzinger, RoudierDaval, \& Khayat, 2006), les études de contexte ont montré que les effets de la défavorisation d'un territoire ne s'observaient pas seulement sur la santé des plus 
défavorisés mais concernaient l'ensemble des habitants de ce territoire. Une étude sur les Zones Urbaines Sensibles observe qu'un ouvrier habitant dans une commune favorisée sera, par exemple, en meilleure santé que celui d'une commune défavorisée (Allonier, Debrand, Lucas-Gabrielli \& Pierre, 2009). Les caractéristiques socioéconomiques ou culturelles d'un territoire doivent donc être reconnues comme des facteurs géographiques à part entière, dans le sens où elles influent localement sur les comportements et les risques de santé. En ce sens, la progression de la fragmentation socio-spatiale, qui est une tendance de l'espace géographique français, peut apparaître prospectivement comme un facteur favorable à la progression des inégalités territoriales de santé. L'impact de la composition socio-économique d'un territoire est depuis longtemps exploré par l'épidémiologie sociale, via notamment les indicateurs de défavorisation socio-économique qui permettent de mesurer le degré de vulnérabilité sociale des populations. Différents indicateurs, plus ou moins récents, ont été produits dans le monde anglo-saxon (Townsend, 1987 ; Carstairs \& Morris, 1989; Raymond \& Pampalon, 2003) et en France (Pornet et al., 2012 ; Rey, Jougla, Fouillet, \& Hémon, 2009). Ils se distinguent essentiellement par l'échelle et le choix des variables agrégées afin de constituer l'indicateur géographique, même si beaucoup d'entre eux utilisent notamment des variables sur le niveau de revenus, le taux de chômage, le niveau d'éducation, la catégorie socio-professionnelle (CSP) ou encore le capital social.

L'influence du territoire sur la santé peut aussi être appréhendée dans sa dimension médicale, tant l'organisation locale et l'accessibilité du système de soins constitue une ressource indispensable à la santé (Tanke \& Ikkersheim, 2012). Il est par exemple constaté un plus faible recours aux soins dans les territoires où la densité médicale est plus faible (Léonard, Stordeur, \& Roberfroid, 2009). D'autres travaux montrent que la distance aux services de santé freine aussi le recours aux soins et contribue ainsi aux inégalités de santé (Korda, Butler, Clements, \& Kunitz, 2007). Pour Bisonnette, la «disponibilité et l'accessibilité aux soins est une des seules caractéristiques territoriales ayant un impact direct sur la santé » (Bissonnette, Wilson, Bell, \& Shah, 2012). En effet, l'équipement médical d'un territoire est susceptible de favoriser à la fois la prévention et le diagnostic précoce des maladies, mais aussi le suivi et la qualité de la prise en charge de ces pathologies et peut se mesurer de différentes manières. La qualité médicale d'un territoire se situe donc dans sa capacité à donner accès aux ressources sanitaires nécessaires à la santé. L'accessibilité des soins dépend à la fois de la disponibilité de l'offre, c'est à dire la quantité et à la localisation des points d'offre par rapport à la demande, et de son accessibilité physique. Cette dernière dépend de la capacité de déplacement du patient entre sa localisation et les points d'offre (Guagliardo, 2004). La notion d'accessibilité tient donc compte de la mobilité des patients et des ressources en transport mobilisables, ainsi que du trajet à parcourir en temps, en distance et en coût. De ce fait, les indicateurs d'accessibilité médicale ne peuvent être calculés uniquement par la présence ou l'absence de ces points d'offre, mais doivent être rapportés aux besoins et à la demande du territoire et des territoires environnants.

\section{Les inégalités territoriales de santé, phénomène complexe}

7 Si les études contextuelles ont globalement cherché à évaluer l'impact spécifique de chaque caractéristique géographique, l'identification de ces déterminants géographiques de la santé n'aide que partiellement à la compréhension des inégalités 
territoriales de santé. Les précédentes expériences sur les déterminants sociaux de la santé (tabagisme, dépistage) montrent qu'agir sur ces déterminants n'aboutit pas nécessairement à une réduction des inégalités. Cette distinction entre la recherche sur les déterminants et celle sur les inégalités de santé était déjà soutenue par Rose à la fin des années 1980: “'Pourquoi certains individus ont de l'hypertension?' est une question assez différente de 'pourquoi certaines populations ont de l'hypertension, alors qu'elle est rare chez d'autres?'. Ces questions demandent différents types d'études et elles ont des réponses différentes " (Rose, 1985). Alors que l'exploration des déterminants géographiques de la santé est déjà bien engagée, il semble nécessaire de s'appuyer sur ces nouvelles connaissances afin d'étudier la capacité du territoire à générer des inégalités de santé.

En effet, géographes et épidémiologistes partagent bien souvent les mêmes outils dans l'analyse géographique des faits de santé, mais la sensibilité de chaque discipline oriente leur interprétation et leur approche des inégalités territoriales de santé. Si la géographie de la santé ne doit pas avoir seulement pour objectif « d'étudier la santé en fonction des lieux mais d'étudier les lieux au regard de la santé, des soins et des politiques de santé » (Fleuret \& Séchet, 2011), l'analyse géographique des inégalités territoriales de santé doit aussi porter sur le processus géographique menant à ces inégalités, afin de comprendre l'implication du territoire dans ce processus. La littérature rapporte que de multiples caractéristiques géographiques sont impliquées dans la constitution des inégalités territoriales. Une interprétation géographique des inégalités territoriales de santé nécessite, de ce fait, une approche non pas fragmentée du territoire mais au contraire globale, focalisée sur l'impact du territoire dans son ensemble et regroupant l'ensemble des caractéristiques géographiques susceptibles d'influencer la santé (Figure 1).

Figure 1 : Modalités d'analyse des inégalités territoriales face aux cancers

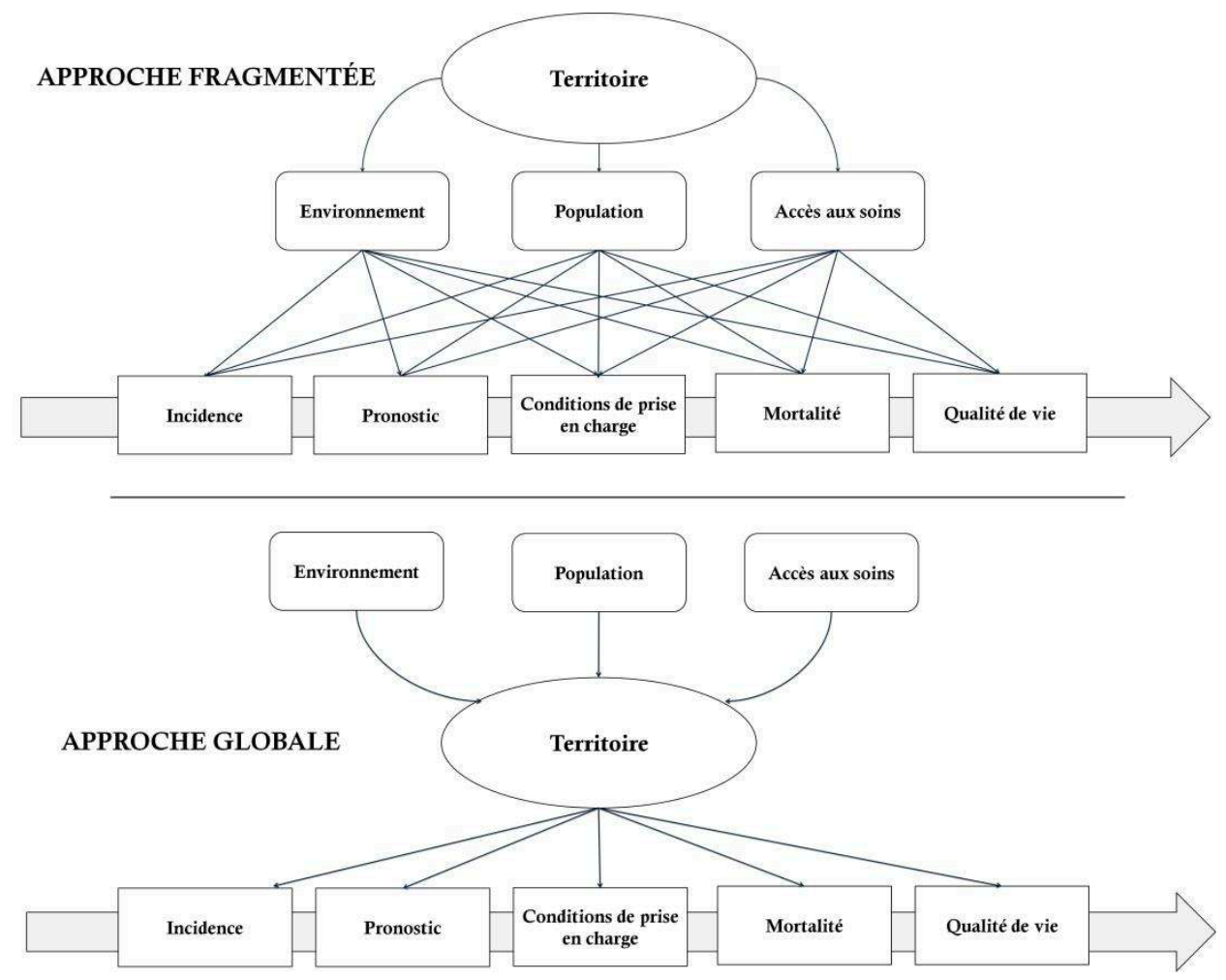


9 L'analyse des inégalités territoriales de santé met en évidence que les territoires, en fonction de certaines caractéristiques, exposent plus ou moins leurs habitants aux risques de santé et peuvent, de ce fait, générer des inégalités entre leurs populations respectives. La constitution d'une typologie territoriale, à partir de ces caractéristiques géographiques reconnues comme influentes sur la santé, permettrait de mettre en évidence la capacité du territoire à générer des inégalités de santé, et de comparer l'état de santé entre des populations différemment exposées par leur contexte de vie. Les typologies territoriales sont déjà fréquemment utilisées en géographie pour donner une lecture synthétique de l'organisation territoriale d'un espace. Une des dernières typologies de l'espace français fait ressortir, à l'échelle du canton, sept profils différents, trois ruraux et quatre plus urbains (Cauchi-Duval \& Bergouignan, 2011). Cette typologie a été construite à partir de quatre catégories de variables : des données sur «l'utilisation des territoires» (logements, emplois), sur «la structure démographique des territoires» (âge, ménages), la "dynamique démographique récente des territoires » et "la structure socio-économique des populations » (CSP, proportion de propriétaires, de chômeurs et d'inactifs). Elle montre le lien de plus en plus marqué entre le parcours résidentiel et la biographie personnelle, comme par exemple avec la situation des diplômés qui se concentrent davantage dans les villes.

10 Plusieurs typologies territoriales ont été réalisées en France pour les études en santé. L'une des premières date de la fin des années 1990 (Lucas-Gabrielli, Tonnelier \& Vigneron, 1998). Réalisée à l'échelle des zones d'emploi, elle intégrait des données sanitaires (offre et consommation de soins, mortalité) et des données socioéconomiques (composition sociale, emploi) sur les territoires, afin d'identifier et de localiser les différents contextes socio-sanitaires en France. Ce type de typologies aide notamment à la prise de conscience, de la part des acteurs publics, de l'hétérogénéité des situations socio-sanitaires en fonction des territoires. Elles montrent aussi la nécessité d'adapter les politiques de santé à ces problématiques locales particulières. Suivant aussi ce principe méthodologique d'agréger des données sur la démographie médicale, l'état de santé et les territoires en eux-mêmes, Trugeon identifie, à l'échelle du canton, « une France en sept classes » (Trugeon, Thomas, Michelot, \& Fédération nationale des observatoires régionaux de santé (France), 2010).

11 D'autres typologies territoriales utilisées pour l'analyse spatiale des faits de santé n'intègrent pas de données sur l'état de santé mais uniquement des variables sur des caractéristiques géographiques reconnues comme ayant une influence sur la santé. On obtient ainsi un découpage géographique "a priori », qui n'est confronté que dans un second temps à des données sanitaires (Gershoff, Pedersen, \& Lawrence Aber, 2009). Par exemple, la typologie de l'aire métropolitaine de Paris, organisée par Van Hulst en fonction de différentes caractéristiques géographiques reconnues dans la littérature comme associées au risque cardio-vasculaire, distingue six profils de territoires, principalement en fonction de leur degré d'urbanisation et de leur composition sociale (Van Hulst et al., 2012). Elle permet ensuite d'observer une pression artérielle significativement plus élevée chez les personnes habitant les quartiers urbains défavorisés, après ajustement sur les facteurs de risques individuels.

12 A l'étranger, Drackley propose une méthodologie relativement similaire, avec l'utilisation d'indicateurs démographiques, sociaux et sur les conditions de logement, dans la municipalité de Peel en Ontario (Drackley, Newbold, \& Taylor, 2011). Arcaya utilise pour sa typologie de l'Etat du Massachusetts 55 variables, réparties en six 
domaines différents: comportements de santé, logement et utilisation du sol, transports, services, composition sociale et composition démographique (Arcaya et al., 2014). Enfin, Weden réalise une typologie territoriale qui pourrait servir à l'étude des inégalités géographiques de santé sur l'ensemble des Etats-Unis (Weden, Bird, Escarce, \& Lurie, 2011). Les données utilisées portent sur les caractéristiques de l'environnement physique (environnement bâti et logement) et de la population (niveau social, origine ethnique et communautés, démographie). Au final, la typologie distingue six «archétypes" de territoires et observe leurs évolutions entre 1990 et 2000. Les auteurs insistent sur la stabilité des effectifs entre 1990 et 2000, qui prouverait la viabilité de cette typologie dans le temps et la capacité du modèle à mesurer l'évolution temporelle des inégalités géographiques de santé.

13 Alors que la cartographie et les études épidémiologiques ne sont pas suffisantes pour comprendre l'implication du territoire dans la constitution des inégalités de santé, le recours à la typologie territoriale permet de constituer un découpage géographique pertinent pour l'analyse des inégalités territoriales de santé, car il synthétise l'ensemble des multiples influences du territoire sur la santé. En distinguant différents profils de territoires en fonction de certaines caractéristiques géographiques connues comme ayant un effet sur la santé des populations, un tel découpage peut permettre d'observer la capacité des territoires à générer des inégalités de santé, puisqu'il comparerait l'état de santé entre des populations différemment exposées aux risques de santé selon leur contexte de vie.

\section{Produire un découpage géographique pertinent pour l'analyse des inégalités territoriales de santé}

14 Afin d'évaluer les possibilités de cette approche méthodologique, une typologie territoriale a été réalisée en Rhône-Alpes. Quinze variables géographiques ont été collectées afin de décrire les dimensions physique, sociale et médicale des territoires en Rhône-Alpes (cf annexe). Cinq variables, disponibles à l'échelle de l'IRIS, peuvent être considérées comme des variables de proximité, fortement sensibles géographiquement, détaillant avec une bonne précision le cadre de vie, la composition sociale du territoire et son niveau d'équipement en commerces et services de proximité. On retrouve ensuite sept variables mesurant des phénomènes locaux, à l'échelle du canton ou de la commune, sur l'environnement, la dynamique foncière, la composition sociale ou encore la démographie médicale. Pour certaines variables, cette échelle a pu être imposée par la non-disponibilité des données à une échelle plus fine, mais aussi par des contraintes méthodologiques. Enfin, trois variables concernent le rapport du territoire au fait urbain et métropolitain, à travers notamment l'accès à un certain nombre d'équipements, et portent sur de entités géographiques étendues comme le bassin de vie ou l'aire urbaine. L'analyse multivariée de ces données géographiques permet de constituer, grâce à une Classification Ascendante Hiérarchique, une typologie territoriale en Rhône-Alpes (Figure 2). Pour les quinze variables géographiques comprises dans l'analyse multivariée, nous avons calculé la moyenne en fonction du type de territoire de manière à mieux les décrire et comprendre leurs spécificités (Figure 7 en annexe). Les différents types de territoires ont été nommés en fonction de ces informations. 
Figure 2 : Typologie territoriale pour les Iris de la Région Rhône-Alpes

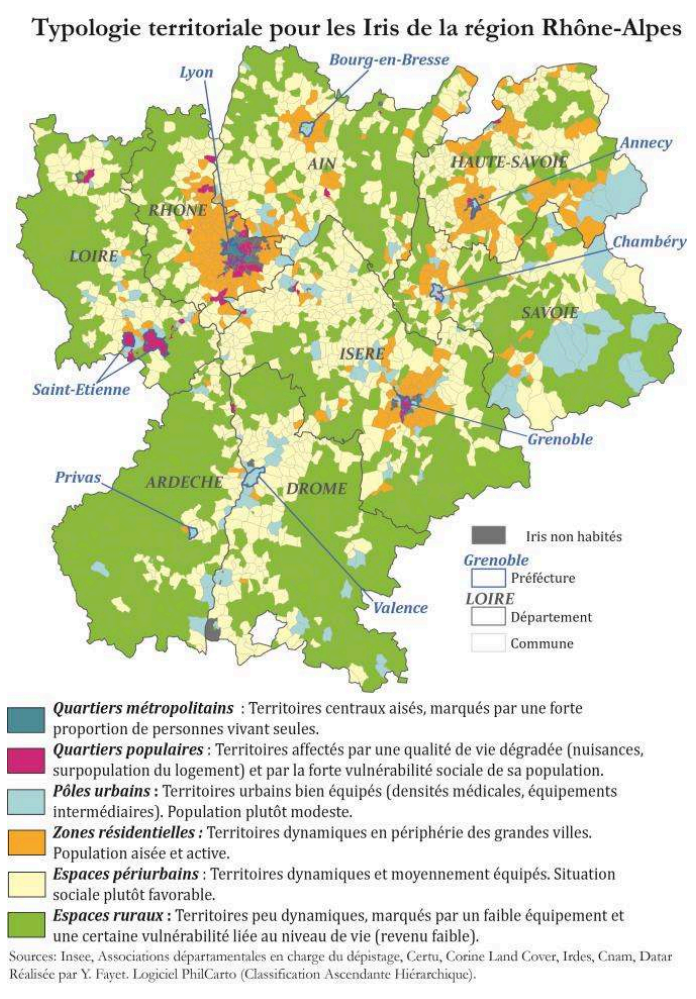

15 Cette typologie distingue d'une part les Quartiers Métropolitains, regroupant les centres villes des deux plus grandes villes de la Région (Lyon, Grenoble), avec une population aisée et une forte proportion de personnes vivant seules. La pression foncière et le niveau de densité de population sur ces territoires se font ressentir sur la qualité d'équipement médical, avec des indicateurs moyens. Les Quartiers Populaires, quant à eux, désignent les Iris des principales agglomérations affectées par une qualité de vie dégradée, du fait de la forte exposition aux nuisances et de la surpopulation du logement, et par la forte vulnérabilité sociale de sa population, avec un revenu médian faible et un taux de chômage élevé.

16 Les pôles urbains sont les autres territoires urbains de la région, qui se distinguent par la qualité de leurs équipements (densités médicales, équipements intermédiaires) et une population plutôt modeste. Enfin, le dernier type de territoire que l'on peut raisonnablement rattaché au fait urbain est celui des zones résidentielles. Ces territoires profitent de leur situation géographique en proche périphérie des grandes villes pour attirer des populations aisées, ayant des revenus élevés, en leur offrant un cadre de vie agréable.

La classe des espaces périurbains rassemble des territoires dynamiques démographiquement, attirant les travailleurs urbains repoussés plus loin en périphérie par le prix du foncier. Ce sont des territoires moyennement équipés où l'on retrouve une situation sociale plutôt favorable, notamment avec une faible part de personnes vivant seules. Enfin, les espaces ruraux sont les territoires les plus en périphérie des grandes villes. Peu dynamiques, ils sont marqués par un très faible équipement, aussi bien pour les services que pour l'équipement médical, et par une certaine vulnérabilité liée au niveau de vie, avec un faible revenu médian. 
L'analyse des données géographiques selon les types de territoires montre d'importants contrastes géographiques entre ces différents contextes de vie (Figure 7 en annexe). Deux types de territoires paraissent particulièrement vulnérables. Les quartiers populaires, tout d'abord, où près d'un quart de la superficie est occupé par des nuisances contre moins de $5 \%$ en moyenne dans la Région avec un revenu médian inférieur d'un tiers à celui perçu par les habitants des zones résidentielles ou des quartiers métropolitains. Les espaces ruraux se distinguent, ensuite, par leur très faible niveau d'équipement, notamment en ce qui concerne les médecins généralistes. On retrouve, en effet, presque trois fois plus d'Equivalents Temps Plein de médecins généralistes dans les pôles urbains, par rapport aux espaces ruraux. Réalisé à l'échelle de l'IRIS, ce découpage permet une analyse géographique fine et statistiquement puissante des inégalités, puisque chaque type de territoire compte, au minimum, 500000 habitants. De plus, les contrastes observés sur la typologie en Rhône-Alpes illustrent bien les bénéfices attendus de cette méthodologie pour l'analyse des inégalités territoriales de santé, puisque la typologie permet de comparer des populations qui ne sont pas du tout exposées aux mêmes risques de santé par leur contexte de vie.

\section{Les inégalites face aux cancers, un processus évolutif}

19 A l'origine de 8,2 millions de décès en 2012, le cancer est considéré par l'OMS comme une "cause majeure" de décès dans le monde. Il constitue la première cause de mortalité en France, puisqu'on l'estime responsable de 148000 décès en 2012 (85 000 hommes, 63000 femmes, données INCa). Alors que les différents indicateurs montrent une progression constante de la survie, marquant ainsi les progrès et le niveau de performance du système de santé français, la persistance voire l'accroissement des inégalités face au cancer signale que ces bénéfices actuels ne profitent pas à tous et qu'ils sont même de moins en moins bien répartis. Ces inégalités révèlent l'existence d'un risque différencié entre différentes populations et signifient donc que des patients, en fonction de leur appartenance à un groupe social ou à un territoire, peuvent connaître des trajectoires cliniques et des issues différentes. Afin de mieux comprendre ce phénomène, une approche méthodologique focalisée sur la reconstitution de ce processus médical discriminant semble nécessaire. Cette reconstitution chronologique s'impose d'autant plus que le pronostic et la guérison de chaque patient atteint de cancer est la conséquence d'un parcours long de plusieurs mois voire d'années, impliquant de multiples facteurs.

\section{Le cancer, maladie au long cours}

L'apparition d'un cancer est souvent le résultat d'un long mécanisme, nécessitant de multiples modifications cellulaires. C'est pourquoi le risque de cancer augmente avec l'âge, en raison de l'accumulation des agressions externes subies par les cellules et, probablement, de la moindre efficacité des mécanismes de réparation de l'ADN chez les personnes âgées. Les autres facteurs de risque des cancers sont principalement liés à l'environnement (rayonnements, virus, expositions industrielles...), aux modes de vie (tabac, alcool, alimentation...) et à l'hérédité. Différentes études montrent que l'impact des facteurs environnementaux dans l'incidence et la mortalité par cancer prévaut sur 
les facteurs de risques génétiques (Czene, Lichtenstein, \& Hemminki, 2002 ; Irigaray et al., 2007). Les découvertes des travaux en épigénétique, montrant l'influence de l'environnement sur l'expression des gènes impliqués dans la cancérogénèse, corroborent aussi cette idée (Esteller, 2008 ; Feinberg \& Tycko, 2004).

Mais les inégalités face au cancer en amont du processus thérapeutique ne sont pas seulement quantitatives, sous formes de disparités d'incidence. Elles peuvent être aussi en lien avec les caractéristiques des patients et de leurs tumeurs, dans le sens où la situation clinique du patient au moment de son diagnostic détermine en partie ses chances de survie. Le stade constitue, par exemple, un indicateur pronostique pertinent puisqu'il permet de connaître le degré d'avancement de la maladie au moment de son diagnostic. Le stade I regroupe les tumeurs de petite taille quand les stades III et IV correspondent à des tumeurs en progression locale (stade III) ou métastatique (stade IV). Par ailleurs, afin d'évaluer le degré de malignité de la tumeur, certains types de cancers peuvent être gradés en fonction de différents critères morphologiques. La classification va généralement du grade I au grade III, voire IV dans certains cas (tumeurs cérébrales), le grade IV désignant les tumeurs les plus agressives et donc de plus mauvais pronostic. En plus des caractéristiques tumorales, les chances de survie du patient sont aussi impactées par son état de santé général au moment du diagnostic. L'âge ou la présence de co-morbidités sont très souvent associés à un plus mauvais pronostic (Jooste et al, 2013 ; Sogaard, Thomsen, Bossen, Sorensen, \& Norgaard, 2013).

Une abondante littérature épidémiologique, en grande majorité anglo-saxonne, fait état d'une inégale répartition de ces différents facteurs de risque pré-thérapeutiques. Il est ainsi prouvé que les personnes issues de milieux défavorisés (Baade, Dasgupta, Aitken, \& Turrell, 2011; Goovaerts \& Xiao, 2012; Henry, Niu, \& Boscoe, 2009) ou habitant dans des territoires à faible densité médicale (Ananthakrishnan, Hoffmann, \& Saeian, 2010 ; Berger et al., 2012 ; Blankart, 2012) sont généralement diagnostiquées plus tardivement, à un stade plus avancé de la maladie. Ces retards aux diagnostics peuvent être liés à une moindre participation de ces populations aux campagnes de dépistage, que ce soit en France (Vallée \& Chauvin, 2012) ou aux Etats-Unis (Barry \& Breen, 2005 ; Hines, Markossian, Johnson, Dong, \& Bayakly, 2014).

Ces résultats signifient, qu'en fonction de clivages sociaux ou géographiques, les populations ne présentent pas toutes les mêmes chances de survie avant leur entrée dans le processus thérapeutique.

La phase de traitement d'un cancer a pour objectif la disparition des cellules tumorales, tout en prévenant le risque de rechute. Les traitements sont souvent distingués en fonction de leur position et leur rôle par rapport à la chirurgie, qui permet l'ablation de la tumeur mais aussi l'établissement ou la confirmation du diagnostic. Les traitements néo-adjuvants sont réalisés avant la chirurgie pour traiter les métastases ou faciliter cette chirurgie en réduisant la masse tumorale. Les traitements adjuvants complètent une chirurgie non optimale et doivent prévenir le risque de rechute. La radiothérapie et/ou la chimiothérapie sont ainsi couramment utilisées, souvent en complément de la chirurgie. Le processus thérapeutique mobilise, très souvent, différents types de traitements qui ont des fonctions différentes et dont les modalités d'usage sont déterminées en fonction de la situation clinique particulière de chaque patient. A cette multiplicité des options et protocoles thérapeutiques s'ajoutent celles des acteurs du soin, selon leur spécialité ou leur niveau d'expertise de la pathologie. 

inégalités face aux cancers. En effet, au-delà des caractéristiques du patient, la qualité de la prise en charge est aussi un facteur déterminant de sa rémission. Cette qualité des soins concerne à la fois la pertinence de la décision médicale, par exemple au moment où le médecin décide d'un protocole de soins, mais aussi de la réalisation même de l'acte thérapeutique qui peut être plus ou moins bien exécuté. La qualité des soins est définie par l'Institut de médecine des Etats-Unis comme «la capacité des services de santé destinés aux individus et aux populations d'augmenter la probabilité d'atteindre les résultats de santé souhaités, en conformité avec les connaissances professionnelles du moment » (Baker, 2001). Dans cette recherche du traitement optimal, le niveau de spécialisation du praticien et de son établissement semblent être un facteur pronostic important, susceptible d'améliorer les chances du patient de recevoir le traitement le plus adéquat.

L'association entre le volume et la qualité des soins, moins évidente lorsqu'il s'agit de traitements peu complexes, est confirmée par la littérature dans le cas des prises en charges oncologiques (Birkmeyer, Sun, Wong, \& Stukel, 2007 ; Hillner, Smith, \& Desch, 2000).

Néanmoins, alors qu'elles constituent «un champ de recherche essentiel pour l'amélioration de la qualité des soins et l'équité du système de soins", les pratiques médicales et leurs conséquences sur les patients atteints de cancer sont encore peu étudiées en France (Chauvin, Ray-Coquard, \& Philip, 2006). Si on dispose encore d'assez peu d'éléments pour analyser les inégalités de prise en charge du cancer en France, la littérature montre déjà certaines inégalités dans l'accès aux centres spécialisés. De par leur expertise et leur volume de soins, ces structures sont principalement localisées dans les plus grandes villes et sont, dès lors, moins accessibles aux patients éloignés des grands centres urbains (Baird, Flynn, Baxter, Donnelly, \& Lawrence, 2008 ; Onega et al., 2008). En France, différentes études issues des Registres français des cancers montrent l'impact de l'éloignement géographique et de la défavorisation socio-économique sur l'accès aux centres spécialisés (Blais, Dejardin, Boutreux, \& Launoy, 2006 ; Gentil et al., 2012 ; Jambon et al., 2010). Cette moindre accessibilité, pour les populations de certains territoires, aux centres spécialisés est susceptible de participer aux inégalités, même si l'on manque aujourd'hui de données disponibles pour le vérifier.

L'objectif du processus thérapeutique est d'obtenir et de maintenir une rémission du cancer, c'est-à-dire une disparition des signes et des symptômes de la maladie. Une fois les traitements terminés, le patient entre alors dans une phase de surveillance qui a pour objectifs de s'assurer de la qualité de vie et de l'état de santé général du patient, de prévenir et traiter les effets indésirables liés aux traitements, et enfin de détecter d'éventuelles signes de récidive pour pouvoir les traiter rapidement. La surveillance peut être assurée par le chirurgien qui a réalisé l'opération, par l'oncologue médical ou par le médecin traitant. On parle de guérison lorsque la durée de la rémission est suffisante pour écarter le risque d'une rechute ultérieure, généralement au bout de cinq ans, parfois plus. Cependant, le protocole de surveillance peut ne pas être correctement suivi et ces impairs peuvent avoir des conséquences importantes sur le pronostic du patient. En effet, comme pour la prise en charge initiale, le diagnostic et la prise en charge précoces de la rechute permettent la mise en place de traitements moins lourds pour le patient et améliorent sensiblement ses chances de survie. 

santé, les effets aussi bien physiques, sociaux ou mentaux de la maladie se font souvent sentir et peuvent largement perturber son retour à une vie "normale». Les chimiothérapies et radiothérapies peuvent aussi entrainer des troubles hormonaux, cardiaques ou rénaux, ainsi qu'une altération de la fertilité (Helg, 2011). On peut aussi parler des effets à long terme sur la fatigue, la douleur, les troubles visuels et auditifs qui se font ressentir sur la qualité de vie de ces personnes alors qu'elles ont quitté le processus de soin (Cella, Lai, Chang, Peterman, \& Slavin, 2002 ; Kangas, Bovbjerg, \& Montgomery, 2008). Ainsi, malgré la rémission voire la guérison, beaucoup de personnes gardent les stigmates de cette maladie. Les conséquences de la prise en charge et de cette expérience du cancer se retrouvent aussi sur la santé mentale du patient, qui peut être confronté à de nouvelles difficultés dans son retour au travail, son insertion sociale ou encore sa vie privée (Dauchy et al., 2013 ; Le Corroller-Soriano, Malavolti, \& Mermilliod, 2008). Ces nouveaux obstacles liés à l'après-cancer ne sont pas relatifs à l'état de santé du patient, mais à différents aspects de sa vie sociale.

\section{Décomposition chronologique des inégalités de mortalité grâce à la cohorte EMS}

Parce que le parcours clinique d'un patient est constitué de nombreuses étapes, potentiellement discriminantes, les inégalités face aux cancers doivent être considérées comme la conséquence d'un processus construit dans le temps, qui pourrait être retracé grâce aux données de santé. Face à ce processus d'inégalités, la simple cartographie des inégalités ou l'analyse des déterminants de l'incidence ou de la survie ne peuvent être suffisantes afin d'explorer la relation entre ces différents temps de la maladie et de comprendre la construction chronologique de ces inégalités. En effet, ces méthodologies classiques ne permettent pas d'identifier les étapes décisives et les facteurs les plus impliqués dans ce processus. Elles ne peuvent pas soutenir l'action des pouvoirs publics à agir avec certitude sur ce qui participe à la construction des inégalités. La décomposition de ce processus permettrait de savoir si les inégalités de mortalité constatées sont le fait d'une incidence plus importante du cancer ou/et d'une moindre survie des patients. De plus, la moindre survie des patients peut s'expliquer par le pronostic défavorable des patients au moment de leur diagnostic ou/et par leurs conditions de prise en charge qui peuvent aggraver ou améliorer ce pronostic initial.

31 Alors que l'on peine à agir efficacement sur les inégalités face aux cancers, la modélisation de ce processus permettrait de connaitre les facteurs décisifs et leur poids dans la construction des inégalités. Moatti soulignait qu'« un diagnostic en vue de la correction d'inégalités en matière de cancers implique un processus de réflexion multiétapes, qui demeure souvent masqué aux yeux même des acteurs sociaux ou des décideurs qui l'émettent » (Moatti, 2008). Une lutte plus efficace contre les inégalités face aux cancers passe par une meilleure compréhension du processus médical aboutissant à ces inégalités, en retraçant, grâce aux données médicales, l'évolution des chances de survie des patients au cours de leur prise en charge. Mais une telle reconstitution s'avère, de façon générale, très difficile à réaliser aujourd'hui, en raison du morcellement des données médicales et cliniques entre les différents acteurs du système de santé.

Revue francophone sur la santé et les territoires , Miscellanées 

depuis leur diagnostic jusqu'à leur période de rémission, permettent de reconstituer ce processus de construction des inégalités de mortalité. Les sarcomes sont des tumeurs rares pouvant apparaître dans toutes les parties du corps à partir de cellules des tissus conjonctifs, alors que l'essentiel des cancers se déclare à partir des cellules épithéliales ou sanguines. Ces cancers peuvent survenir à des âges plus jeunes comparativement à d'autres cancers. Avec une incidence estimée à 6/100 000 habitants, ils touchent environ 4000 personnes par an en France, ce qui représente moins de $2 \%$ de l'ensemble des tumeurs malignes de l'adulte (Mastrangelo et al., 2012). De nombreuses études épidémiologiques de type cas-témoins ont tenté de définir des facteurs de risque, en particulier dans les populations pédiatriques, mais peu ont retrouvé un lien statistiquement significatif et les résultats sont souvent contradictoires (Clark, Fisher, Judson, \& Thomas, 2005 ; Penel, Pasquier, \& Vanseymortier, 2007). Seuls quelques facteurs de risque comme certaines maladies génétiques (syndrome de Li Fraumeni, neurofibromatose, rétinoblastome héréditaire, ...) et certains facteurs iatrogènes (radiations, chlorure de vinyle), pouvant expliquer $10 \%$ des cas, sont aujourd'hui avérés. D'autres facteurs environnementaux sont suspectés, comme les pesticides et certaines infections virales, mais il est souvent difficile de mesurer l'exposition à ces facteurs et les études pluridisciplinaires doivent être poursuivies. cellules) différents et plus de 150 sous-types moléculaires. L'exérèse chirurgicale est le traitement privilégié en première ligne et le pronostic du patient est largement associé à cette qualité de prise en charge initiale (Blay et al., 2017 ; Derbel et al., 2012 ; Rossi et al., 2013). La méconnaissance et la complexité de ces tumeurs nécessitent une prise en charge initiale planifiée, coordonnée et spécialisée de façon à garantir au maximum les chances de survie des patients. Il est, par exemple, recommandé par l'Institut National du Cancer que la stratégie thérapeutique de tout patient diagnostiqué pour un sarcome soit discuté dans une des RCP (Réunions de Concertation Pluridisciplinaire) dédiées aux sarcomes et labélisées depuis 2010.

Le programme de recherche EMS avait pour objectif d'évaluer la prise en charge des patients atteints de sarcome de façon prospective et exhaustive, d'évaluer la conformité de la prise en charge par rapport aux référentiels de pratiques médicales existants et d'identifier des prises en charges optimales. La cohorte EMS ( $n=698)$ regroupe tous les nouveaux patients diagnostiqués pour un sarcome en 2005 et 2006 (récidives exclues) et habitant en Rhône-Alpes au moment du diagnostic. Les modalités de constitution de la cohorte EMS ont fait l'objet de précédentes publications (Ducimetiere et al., 2010; Lurkin et al., 2010). Les caractéristiques tumorales et cliniques du patient, son adresse au diagnostic, les différents examens et séquences de traitement ainsi que la surveillance post-thérapeutique ont été collectés pour chacun d'entre eux, au sein de leurs différents établissements de prise en charge. L'ensemble du parcours de soins de chaque patient de la cohorte EMS est donc renseigné, depuis le diagnostic initial jusqu'à la dernière visite de surveillance ou au décès. Une première analyse géographique de ces données montrait une incidence plus importante dans les territoires au Nord-Est de la région et une inégale répartition géographique selon les sous-types (Fayet et al., 2014). L'analyse des parcours de soins soulignait aussi que trois caractéristiques cliniques relatives au patient (la localisation, l'âge, le grade) et un 
critère de prise en charge initiale (présence de biopsie) influençaient significativement la distance parcourue par le patient pour son traitement en première ligne.

L'exhaustivité de la cohorte EMS donne la possibilité d'analyser la construction des inégalités territoriales de mortalité pour les patients atteints de sarcomes en RhôneAlpes. Cela nécessite de comparer la situation de chaque type de territoire en fonction d'indicateurs représentatifs des différents temps de la maladie. De façon générale, les inégalités de mortalité traduisent l'action successive de trois autres inégalités précédentes (cf Figure 1):

- des inégalités d'incidence car la mortalité est d'autant plus forte que le nombre de malades est important

- des inégalités pronostiques car la mortalité est d'autant plus forte que les chances de survie des patients au moment de leur diagnostic sont faibles

- des inégalités de prise en charge car la mortalité est d'autant plus forte que la prise en charge du patient est de moindre qualité

Alors que le calcul et l'utilisation de taux d'incidence et de mortalité est assez courant, il semble plus difficile de mesurer, par un indicateur quantitatif synthétique, la qualité de leur prise en charge des patients. Le pronostic initial (au moment de son diagnostic) des patients peut lui être estimé, grâce à un modèle de régression logistique regroupant les différents facteurs pré-thérapeutiques reconnus comme ayant une influence directe sur le pronostic. Pour la cohorte EMS, différents facteurs pronostiques pour le patient atteint de sarcome ont été intégrés dans le modèle statistique, à savoir l'âge du patient au moment du diagnostic, le fait que ce patient soit diagnostiqué ou non à un stade avancé (stades 3 et 4 ), ainsi que le type histologique (GIST, liposarcomes, sarcomes inclassés, sarcomes osseux, autres sarcomes) de son sarcome. Une régression logistique a permis d'évaluer l'influence de ces différents facteurs pronostiques sur le risque de décès à cinq ans pour l'ensemble de la cohorte EMS, ce qui a permis ensuite d'estimer le pronostic de chaque patient à partir de ses propres caractéristiques. Le pronostic des patients au moment de leur diagnostic a donc été estimé sous la forme d'un risque relatif de décès à cinq ans, qui est en moyenne de 0,358 pour l'ensemble des patients de la cohorte EMS. Pour vérifier la qualité de notre calcul, les patients de la cohorte EMS ont été répartis en cinq quintiles selon leur risque initial de décès puis nous avons calculé la mortalité à cinq ans pour chacun de ces quintiles. Seulement $7 \%$ des patients classés, au moment du diagnostic, comme étant de «bon pronostic » sont décédés cinq ans plus tard. A l'inverse, ce risque de décès est de $80 \%$ chez les patients présentant les pronostics les plus défavorables. Ces résultats montrent la pertinence et la qualité prédictive de cette estimation probabiliste et individuelle du pronostic.

Chaque patient de la cohorte EMS a été localisé grâce à l'adresse enregistrée au moment du diagnostic, afin d'établir son IRIS de résidence. Les patients ont ensuite été regroupés selon leur type de territoire. Grâce au recensement de tous les diagnostics de sarcomes en Rhône-Alpes sur deux ans ainsi que le suivi à cinq ans, des taux bruts d'incidence et de mortalité ont été calculés en rapportant le nombre de diagnostics et de décès à la population totale de chaque type de territoire. Afin d'observer l'impact des inégalités pronostiques et de prise en charge sur les inégalités de mortalité, nous avons calculé un taux de mortalité théorique, obtenu en multipliant le taux d'incidence par le risque initial de décès. Pondérant l'incidence en fonction du plus ou moins bon pronostic des populations, cet indicateur est une estimation, réalisée au moment du diagnostic, de la mortalité que l'on pourrait attendre cinq ans plus tard si tous les 
patients avaient les mêmes chances de survie au cours de leur prise en charge. La comparaison de la mortalité théorique avec la mortalité effectivement observée cinq ans plus tard permet d'observer l'évolution des inégalités durant la période thérapeutique. Par exemple, une mortalité observée supérieure à la mortalité théorique signifierait que la population en question a perdu des chances de survie durant sa prise en charge par rapport à la moyenne régionale.

Les variables représentatives des différents temps du cancer (incidence, risque initial de décès, mortalité théorique, taux de décès, mortalité) ont été transposées sous forme d'indices, en prenant la moyenne régionale comme référence (Rhône-Alpes $=100$ ). Ce calcul d'indice permet de pouvoir comparer l'évolution du risque selon le type de territoire par rapport à la moyenne régionale et de mieux comprendre la construction des inégalités territoriales face aux cancers.

\section{Identifier les pertes de chances spécifiques à chaque territoire}

L'analyse géographique des données de la cohorte EMS montre, tout d'abord, que les sarcomes surviennent plus fréquemment dans les pôles urbains, alors que le plus faible taux d'incidence se trouve dans les quartiers métropolitains (Figure 3).

Figure 3 : Inégalités géographiques pour les patients diagnostiqués pour un sarcome en RhôneAlpes en 2005 et 2006

\begin{tabular}{|c|c|c|c|c|c|c|c|}
\hline Variables & $\begin{array}{c}\text { Quartiers } \\
\text { métropolitains }\end{array}$ & $\begin{array}{l}\text { Quartiers } \\
\text { populaires }\end{array}$ & Pôles urbains & $\begin{array}{c}\text { Zones } \\
\text { résidentielles }\end{array}$ & $\begin{array}{l}\text { Espaces } \\
\text { périurbains }\end{array}$ & $\begin{array}{l}\text { Espaces } \\
\text { ruraux }\end{array}$ & $\begin{array}{l}\text { Rhône- } \\
\text { Alpes }\end{array}$ \\
\hline $\begin{array}{l}\text { Taux d'incidence brut } \\
\text { (pour } 100000 \text { habitants) }\end{array}$ & 4,88 & 5,95 & 6,96 & 6,17 & 5,44 & 5,21 & 5,83 \\
\hline Taux d'incidence (indice) & 83,7 & 102,0 & 119,3 & 105,9 & 93,4 & 89,3 & 100 \\
\hline $\begin{array}{c}\text { Risque initial de décès à } 5 \text { ans } \\
\text { (risque relatif) }\end{array}$ & 0,40 & 0,36 & 0,40 & 0,30 & 0,33 & 0,39 & 0,36 \\
\hline $\begin{array}{l}\text { Taux de mortalité théorique } \\
\text { (pour } 100000 \text { habitants) }\end{array}$ & 1,97 & 2,13 & 2,77 & 1,87 & 1,80 & 2,02 & 2,09 \\
\hline $\begin{array}{l}\text { Taux de mortalité théorique } \\
\text { (indice) }\end{array}$ & 94,2 & 102,1 & 132,7 & 89,8 & 86,0 & 96,9 & 100 \\
\hline Décès à 5 ans (\%) & 36,7 & 40,7 & 36,6 & 31,8 & 30,0 & 42,7 & 35,8 \\
\hline $\begin{array}{l}\text { Taux de mortalité brute } \\
\text { (pour } 100000 \text { habitants) }\end{array}$ & 1,79 & 2,42 & 2,54 & 1,96 & 1,63 & 2,22 & 2,09 \\
\hline Taux de mortalité (indice) & 85,8 & 116,0 & 121,8 & 94,1 & 78,2 & 106,5 & 100 \\
\hline
\end{tabular}

Par contre, les patients de ces quartiers métropolitains et des pôles urbains présentent un moins bon pronostic au moment du diagnostic, à l'inverse des patients des zones résidentielles qui présentent un risque initial de décès à 5 ans plus faible. Cela s'explique notamment par le fait qu'on observe significativement moins de stades avancés chez ces derniers par rapport au reste de la région $(\mathrm{p}=0,012)$. Ce bon pronostic initial favorise la survie de ces patients des zones résidentielles qui présentent, avec les patients des espaces périurbains, les taux de décès à 5 ans les plus faibles de la Région, au contraire des patients des quartiers populaires et des espaces ruraux. Enfin, l'analyse comparée des taux de mortalité montre une situation globalement plus favorable dans les espaces périurbains tandis que les pôles urbains sont les territoires les plus touchés par la mortalité par sarcomes. 
41 La comparaison graphique des indices de risque initial et de décès confirme l'idée d'une évolution des inégalités territoriales au cours de la prise en charge. En effet, si les patients des quartiers métropolitains et des pôles urbains étaient plus à risque au moment de leur diagnostic, on peut constater que leurs taux de décès sont très proches de la moyenne régionale (Figure 4). La courbe descendante signifie donc que le risque de décès s'est atténué pour ces patients au cours de leur prise en charge, probablement en raison de la qualité des traitements reçus.

42 A l'inverse, les courbes ascendantes en rouge et vert signifient que le risque de décès s'est particulièrement accru pour les patients des espaces ruraux et des quartiers populaires.

Figure 4 : Risque initial de décès et taux de décès selon les types de territoires en Rhône-Alpes

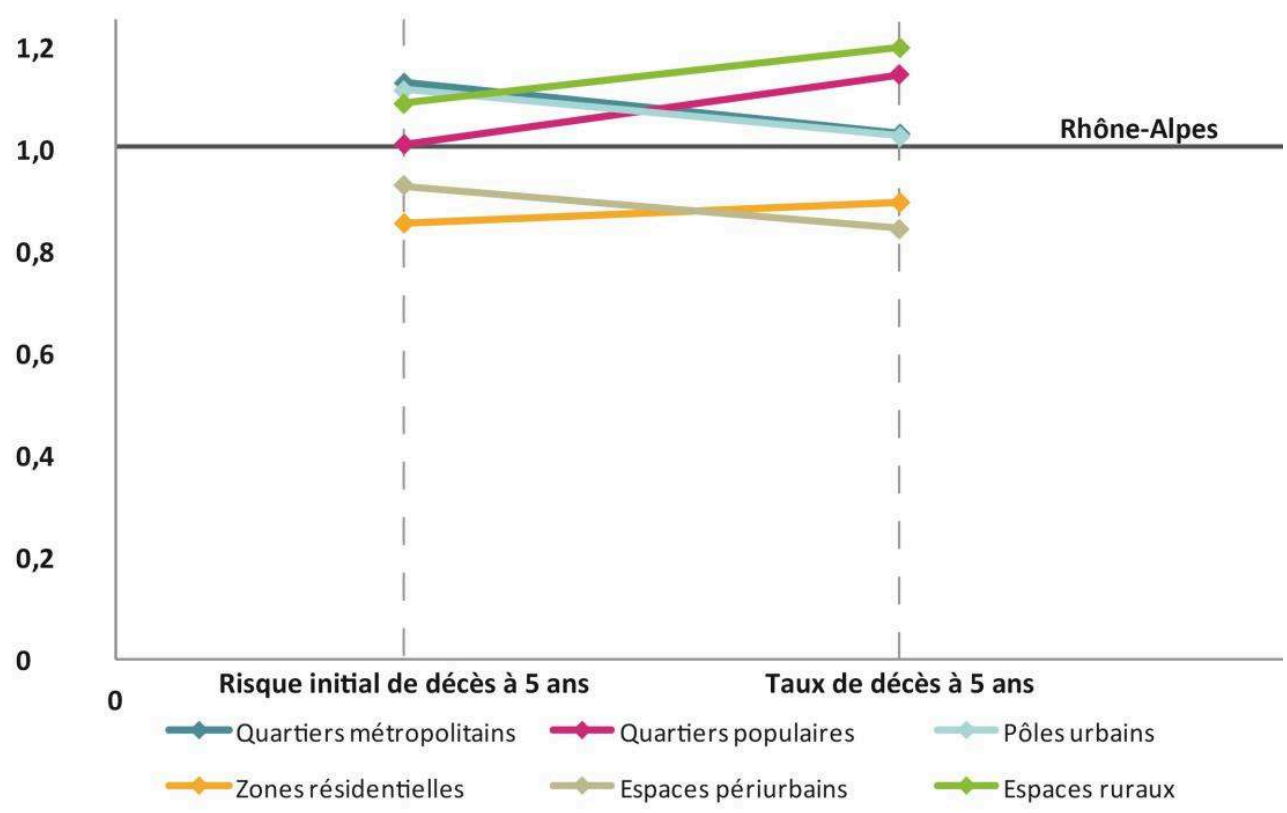

Comme le pronostic initial dans les quartiers populaires était quasiment équivalent à celui de la moyenne régionale, on peut établir que ces pertes de chances se sont produites durant la période thérapeutique. Dans le cas des espaces ruraux, ces pertes de chances survenues au cours de la prise en charge ont amplifié celles observées en amont, puisque les patients ruraux présentaient déjà un pronostic plus défavorable au moment de leur diagnostic.

Ces premiers résultats montrent que, dans le cas des patients diagnostiqués pour un sarcome en Rhône-Alpes en 2005 et 2006, les inégalités territoriales de mortalité ne traduisent pas un gradient territorial bien établi dans le temps. Il semble davantage que ces inégalités résultent d'un processus complexe où les patients de chaque type de territoire sont exposés à des risques spécifiques selon leur contexte de vie. Le décryptage de ces processus géographiquement différenciés nécessite de modéliser l'évolution chronologique du risque dans chaque type de territoire. La comparaison des indices d'incidence, de mortalité théorique et de mortalité observée permet de nous éclairer sur la façon dont se construisent les inégalités territoriales de mortalité. 
Figure 5 : Indices d'incidence, de mortalité théorique et de mortalité selon les types de territoires en Rhône-Alpes

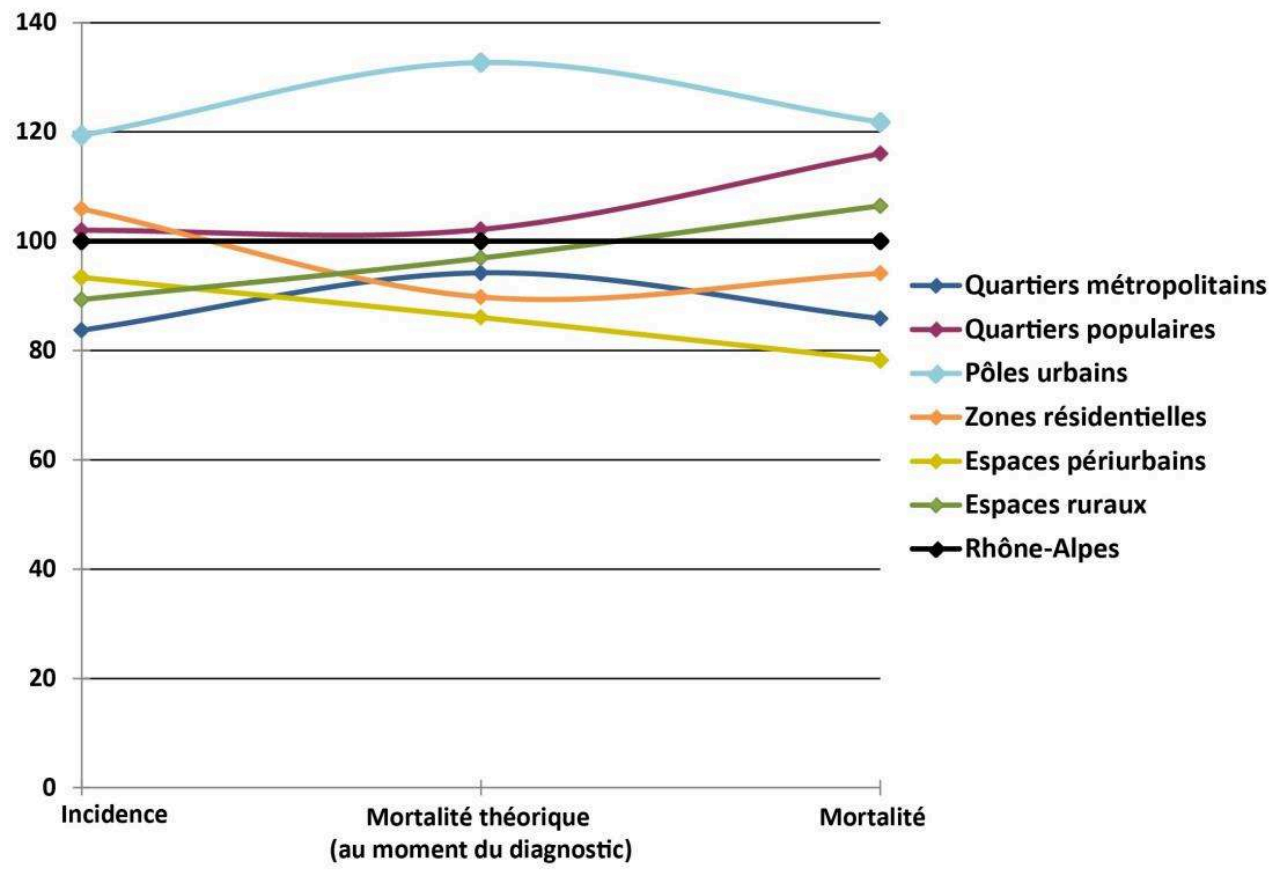

La Figure 5 illustre la diversité des situations et des mécanismes aboutissant aux inégalités territoriales de mortalité. En effet, la surmortalité observée dans trois types de territoires peut être attribuée, grâce à cette modélisation, à trois phénomènes différents. Dans le cas des pôles urbains, la surmortalité est liée essentiellement à la forte surincidence des sarcomes dans ces territoires. En effet, en dépit du fait que ces populations présentaient un risque pronostic élevé (caractérisé par la courbe ascendante entre l'incidence et la mortalité théorique), il a été montré dans le graphique précédent que ces patients "gagnaient» des chances de survie durant la période thérapeutique et présentaient donc des taux de survie sensiblement équivalents à la moyenne régionale. La courbe ascendante puis descendante des pôles urbains traduit bien cette évolution positive des chances de survie durant la prise en charge. Cela reste néanmoins insuffisant pour faire baisser significativement la mortalité, compte tenu de la forte incidence des sarcomes dans ces territoires par rapport au reste de la région.

On constate aussi une mortalité élevée dans les quartiers populaires alors que les indices d'incidence et de mortalité théorique étaient pourtant assez proches de la moyenne régionale. Ces pertes de chances de survie durant la période thérapeutique reflètent probablement des parcours de soins moins optimaux, que ce soit en termes de qualité des soins, d'orientation et de compliance des patients. Enfin, en ce qui concerne les espaces ruraux, la courbe croissante montre que, malgré la deuxième plus faible incidence régionale, le mauvais pronostic et la moindre efficacité des prises en charge engendrent, dans ces territoires, une mortalité supérieure à celle qu'on observe en Rhône-Alpes. Ces courbes permettent aussi d'expliquer la sous-mortalité des zones résidentielles (où le bon pronostic des patients se traduit par une faible mortalité théorique), des quartiers métropolitains (en raison d'une faible incidence et d'une 
meilleure efficacité des prises en charge) et des espaces périurbains (qui cumulent les bons indicateurs).

\section{Discussion}

L'analyse géographique de la cohorte EMS vise à mettre en œuvre deux principes méthodologiques pouvant permettre de mieux comprendre la construction des inégalités territoriales face aux cancers.

D'une part, la réalisation d'une typologie territoriale, reflétant les multiples influences du territoire sur la santé, permet de constituer un découpage géographique pertinent pour l'étude des inégalités territoriales de santé. Ce découpage distingue, en effet, des populations qui sont différemment exposées aux risques de santé de par les caractéristiques de leur contexte de vie. La typologie calculée en Rhône-Alpes permet de montrer d'importants écarts en termes d'expositions aux nuisances environnementales, de défavorisation sociale ou encore d'accès aux soins entre les différents types de territoire. Cette typologie pourrait néanmoins être améliorée par l'intégration de données complémentaires afin de prendre en compte l'ensemble des caractéristiques géographiques reconnues comme ayant un impact sur la santé. On peut penser, par exemple, à des données géographiques sur différentes expositions environnementales (eau, air, sol,...) ou sur la démographie hospitalière qui ne sont pas encore exhaustives ou disponibles en Rhône-Alpes.

De plus, la reconstitution du processus médical menant aux inégalités territoriales de mortalité permet de décomposer le risque et d'identifier plus précisément les étapes décisives dans ce processus. Complétant les approches cartographique et épidémiologique, cette modélisation montre qu'en dépit du poids de la prise en charge thérapeutique dans le vécu du cancer, les inégalités territoriales face aux cancers débutent bien en amont du diagnostic. Compte tenu des séquelles physiques, psychiques et sociales occasionnées par la maladie, on peut aussi facilement imaginer que ces inégalités territoriales se poursuivent bien après la fin des traitements, dans le quotidien souvent contrarié des survivants. L'influence du territoire sur les patients atteints de cancers peut donc être évaluée par le biais de données cliniques mais doit aussi être appréhendée par le prisme du bien-être et de la qualité de vie des patients pendant et suite à leur prise en charge.

Appliquant ces deux principes méthodologiques, nos résultats suggèrent que les inégalités territoriales de mortalité face aux cancers ne traduisent pas un gradient territorial immuable mais résultent, plus certainement, de processus médicaux différenciés selon les types de territoires. On peut constater, en effet, l'évolution spécifique du risque dans chaque territoire suivant les différentes étapes de la maladie. De plus, des territoires en surmortalité, qu'une carte regrouperait logiquement avec le même code couleur, se distinguent pourtant par les processus médicaux à l'origine de cette surmortalité. Dans le cas des sarcomes en Rhône-Alpes, la surmortalité dans les pôles urbains est à mettre au crédit de la forte incidence de ces cancers dans ces territoires, alors que celle des quartiers populaires s'explique par les fortes pertes de chances de survie durant la prise en charge.

51 La méthodologie présentée permet d'observer la construction des inégalités territoriales face aux cancers et d'identifier pour chaque territoire les facteurs responsables de la surmortalité. Suivant le principe de territorialisation des politiques 
de santé, cette méthodologie constitue un outil d'aide à la décision, favorisant la mise en place d'un plan d'actions ciblé et adapté à chaque contexte géographique et à ses risques spécifiques. Dans le cas des sarcomes, notre analyse recommanderait par exemple de s'intéresser à la gestion de la rechute dans les espaces ruraux, à la prise en charge initiale dans les quartiers populaires, ou encore à la prévention dans les pôles urbains, puisqu'on y constate à la fois une forte incidence et des facteurs pronostics plus défavorables. Les progrès réalisés et attendus sur la disponibilité des données de santé pourraient permettre à terme de reproduire cette méthodologie et de répondre aux problématiques de cancers plus fréquents.

\section{Conclusion}

L'analyse géographique de la cohorte EMS s'appuie sur une méthodologie interdisciplinaire, mixant les outils et les concepts utilisés en géographie et en cancérologie. La réalisation d'une typologie territoriale multidimensionnelle, distinguant des populations différemment exposées aux risques de santé selon leur contexte de vie, ainsi que l'analyse de différentes données cliniques, estimant le pronostic initial des patients, ont permis de modéliser la complexe construction des inégalités territoriales de mortalité. Cette analyse comparée de l'évolution du risque met en évidence, dans le cas des sarcomes, l'origine diverse de la surmortalité observée dans trois types de territoires (pôles urbains, quartiers populaires, espaces ruraux). La méthodologie présentée semble donc en mesure de fournir des connaissances précises sur les facteurs participant aux inégalités de mortalité face aux cancers et de proposer des mesures adaptées, pouvant cibler le ou les facteurs impliqués dans chaque contexte géographique. Elle peut ainsi aider à lutter plus efficacement contre les inégalités territoriales face aux cancers et contribuer à la territorialisation des politiques de santé, en se focalisant sur les risques sanitaires spécifiques à chaque territoire.

\section{BIBLIOGRAPHIE}

Allonier C, Debrand T, Lucas-Gabrielli V \& Pierre A (2009). Contexte géographique et état de santé de la population: de l'effet ZUS aux effets de voisinage. Questions d'économie de la santé, 139.

Ananthakrishnan, A. N., Hoffmann, R. G., \& Saeian, K. (2010). S1006 Higher Physician Density is Associated With Lower Incidence of Late-Stage Colorectal Cancer. Gastroenterology, 138(5, Supplement 1), S-159. https://doi.org/10.1016/S0016-5085(10)60728-7

Arcaya, M., Reardon, T., Vogel, J., Andrews, B. K., Li, W., \& Land, T. (2014). Tailoring communitybased wellness initiatives with latent class analysis--Massachusetts Community Transformation Grant projects. Preventing Chronic Disease, 11, E21. https://doi.org/10.5888/pcd11.130215

Baade, P. D., Dasgupta, P., Aitken, J., \& Turrell, G. (2011). Geographic remoteness and risk of advanced colorectal cancer at diagnosis in Queensland: a multilevel study. British Journal of Cancer, 105(7), 1039-1041. https://doi.org/10.1038/bjc.2011.356 
Baird, G., Flynn, R., Baxter, G., Donnelly, M., \& Lawrence, J. (2008). Travel time and cancer care: an example of the inverse care law? Rural and Remote Health, 8(4), 1003.

Baker, A. (2001). Crossing the Quality Chasm: A New Health System for the 21st Century. BMJ, 323(7322), 1192. https://doi.org/10.1136/bmj.323.7322.1192

Barry, J., \& Breen, N. (2005). The importance of place of residence in predicting late-stage diagnosis of breast or cervical cancer. Health \& Place, 11(1), 15-29. https://doi.org/10.1016/ j.healthplace.2003.12.002

Berger, F., Doussau, A., Gautier, C., Gros, F., Asselain, B., \& Reyal, F. (2012). Impact du statut socioéconomique sur la gravité du diagnostic initial de cancer du sein. Revue d'Épidémiologie et de Santé Publique, 60(1), 19-29. https://doi.org/10.1016/j.respe.2011.08.066

Birkmeyer, J. D., Sun, Y., Wong, S. L., \& Stukel, T. A. (2007). Hospital Volume and Late Survival After Cancer Surgery. Annals of Surgery, 245(5), 777-783. https://doi.org/10.1097/01.sla. 0000252402.33814.dd

Bissonnette, L., Wilson, K., Bell, S., \& Shah, T. I. (2012). Neighbourhoods and potential access to health care: The role of spatial and aspatial factors. Health \& Place, 18(4), 841-853. https://doi.org/ 10.1016/j.healthplace.2012.03.007

Blais, S., Dejardin, O., Boutreux, S., \& Launoy, G. (2006). Social determinants of access to reference care centres for patients with colorectal cancer - A multilevel analysis. European Journal of Cancer, 42(17), 3041-3048. https://doi.org/10.1016/j.ejca.2006.06.032

Blankart, C. R. (2012). Does healthcare infrastructure have an impact on delay in diagnosis and survival? Health Policy, 105(2-3), 128-137. https://doi.org/10.1016/j.healthpol.2012.01.006

Blay, J.-Y., Soibinet, P., Penel, N., Bompas, E., Duffaud, F., Stoeckle, E., ... NETSARC/RREPS and French Sarcoma Group-Groupe d'Etude des Tumeurs Osseuses (GSF-GETO) networks. (2017). Improved survival using specialized multidisciplinary board in sarcoma patients. Annals of Oncology: Official Journal of the European Society for Medical Oncology, 28(11), 2852-2859. https:// doi.org/10.1093/annonc/mdx484

Cabidoche, Y.-M., Achard, R., Cattan, P., Clermont-Dauphin, C., Massat, F., \& Sansoulet, J. (2009). Long-term pollution by chlordecone of tropical volcanic soils in the French West Indies: a simple leaching model accounts for current residue. Environmental Pollution (Barking, Essex: 1987), 157(5), 1697-1705. https://doi.org/10.1016/j.envpol.2008.12.015

Carstairs V \& Morris R (1989). Deprivation: explaining differences in mortality between Scotland and England and Wales. British Medical Journal, 299:886-889.

Cauchi-Duval, N., \& Bergouignan, C. (2011). Les spécificités démo-géographiques des territoires en France métropolitaine : analyse de l'évolution des critères de différenciation pour une typologie synthétique. Espace populations sociétés. Space populations societies, (2011/3), 425-439. https://doi.org/10.4000/eps.4635

Cella, D., Lai, J.-S., Chang, C.-H., Peterman, A., \& Slavin, M. (2002). Fatigue in cancer patients compared with fatigue in the general United States population. Cancer, 94(2), 528-538. https:// doi.org/10.1002/cncr.10245

Cervero, R., \& Kockelman, K. (1997). Travel demand and the 3Ds: Density, diversity, and design. Transportation Research Part D: Transport and Environment, 2(3), 199-219. https://doi.org/ 10.1016/S1361-9209(97)00009-6

Chaix, B., Bean, K., Daniel, M., Zenk, S. N., Kestens, Y., Charreire, H., ... Pannier, B. (2012).

Associations of supermarket characteristics with weight status and body fat: a multilevel analysis 
of individuals within supermarkets (RECORD study). PloS one, 7(4), e32908. https://doi.org/ 10.1371/journal.pone.0032908

Chauvin, F., Ray-Coguard, I., \& Philip, T. (2006). Evaluation des pratiques médicales en cancérologie : l'apport des réseaux de soins. Risques \& qualité en milieu de soins, (2), 89-94.

Clark, M. A., Fisher, C., Judson, I., \& Thomas, J. M. (2005). Soft-tissue sarcomas in adults. The New England Journal of Medicine, 353(7), 701-711. https://doi.org/10.1056/NEJMra041866

Czene, K., Lichtenstein, P., \& Hemminki, K. (2002). Environmental and heritable causes of cancer among 9.6 million individuals in the Swedish Family-Cancer Database. Int.J.Cancer, 99(2), 260-266.

Dauchy, S., Ellien, F., Lesieur, A., Bezy, O., Boinon, D., Chabrier, M., ... Seigneur, E. (2013). Quelle prise en charge psychologique dans l'après-cancer ? : Les recommandations de la Société Française de Psycho-Oncologie (SFPO). Psycho-Oncologie, 7(1), 4-17. https://doi.org/10.1007/ s11839-013-0409-3

Diez Roux, A. V., \& Mair, C. (2010). Neighborhoods and health. Annals of the New York Academy of Sciences, 1186(1), 125-145. https://doi.org/10.1111/j.1749-6632.2009.05333.x

Drackley, A., Newbold, K. B., \& Taylor, C. (2011). Defining Socially-Based Spatial Boundaries in the Region of Peel, Ontario, Canada. International Journal of Health Geographics, 10(1), 38. https:// doi.org/10.1186/1476-072X-10-38

Ducimetiere, F., Lurkin, A., Ranchere-Vince, D., Decouvelaere, A. V., Isaac, S., Claret-Tournier, C., ... Ray-Coquard, I. (2010). [Incidence rate, epidemiology of sarcoma and molecular biology. Preliminary results from EMS study in the Rhone-Alpes region]. Bull.Cancer, 97(6), 629-641.

Ellen, I. G., Mijanovich, T., \& Dillman, K.-N. (2001). Neighborhood Effects on Health: Exploring the Links and Assessing the Evidence. Journal of Urban Affairs, 23(3-4), 391-408. https://doi.org/ $10.1111 / 0735-2166.00096$

Esteller, M. (2008). Epigenetics in cancer. The New England Journal of Medicine, 358(11), 1148-1159. https://doi.org/10.1056/NEJMra072067

Fayet, Y., Chasles, V., Ducimetière, F., Collard, O., Berger, C., Meeus, P., ... Ray-Coquard, I. (2014). Répondre aux enjeux des cancers rares. Approche géographique de la cohorte EMS des sarcomes en région Rhône-Alpes. Bulletin du Cancer, 101(2), 127-136. https://doi.org/10.1684/bdc.2014.1891

Feinberg, A. P., \& Tycko, B. (2004). The history of cancer epigenetics. Nature Reviews. Cancer, 4(2), 143-153. https://doi.org/10.1038/nrc1279

Fleuret, S., \& Séchet, R. (2011). Spatialité des enjeux de pouvoir et des inégalités: pour une géographie sociale de la santé. In Penser et faire la géographie sociale : contributions pour une épistémologie de la géographie sociale (p. 333-351). Rennes: Presses Universitaires de Rennes.

Gentil, J., Dabakuyo, T. S., Ouedraogo, S., Poillot, M.-L., Dejardin, O., \& Arveux, P. (2012). For patients with breast cancer, geographic and social disparities are independent determinants of access to specialized surgeons. A eleven-year population-based multilevel analysis. BMC Cancer, 12(1), 351. https://doi.org/10.1186/1471-2407-12-351

Gershoff, E. T., Pedersen, S., \& Lawrence Aber, J. (2009). Creating neighborhood typologies of GISbased data in the absence of neighborhood-based sampling: a factor and cluster analytic strategy. Journal of Prevention \& Intervention in the Community, 37(1), 35-47. https://doi.org/ $10.1080 / 10852350802498458$

Gibson, M., Petticrew, M., Bambra, C., Sowden, A. J., Wright, K. E., \& Whitehead, M. (2011). Housing and health inequalities: A synthesis of systematic reviews of interventions aimed at 
different pathways linking housing and health. Health \& Place, 17(1), 175-184. https://doi.org/ 10.1016/j.healthplace.2010.09.011

Goovaerts, P., \& Xiao, H. (2012). The impact of place and time on the proportion of late-stage diagnosis: The case of prostate cancer in Florida, 1981-2007. Spatial and Spatio-temporal Epidemiology, 3(3), 243-253. https://doi.org/10.1016/j.sste.2012.03.001

Guagliardo, M. F. (2004). Spatial accessibility of primary care: concepts, methods and challenges. International Journal of Health Geographics, 3(1), 3. https://doi.org/10.1186/1476-072X-3-3

Helg, C. (2011). Séquelles incapacitantes après chimio-et radiothérapie. Douleur et Analgésie, 24(S1), 29-33. https://doi.org/10.1007/s11724-011-0250-z

Henry, K. A., Niu, X., \& Boscoe, F. P. (2009). Geographic disparities in colorectal cancer survival. International Journal of Health Geographics, 8, 48. https://doi.org/10.1186/1476-072X-8-48

Hillner, B. E., Smith, T. J., \& Desch, C. E. (2000). Hospital and physician volume or specialization and outcomes in cancer treatment: importance in quality of cancer care. Journal of Clinical Oncology: Official Journal of the American Society of Clinical Oncology, 18(11), 2327-2340.

Hines, R., Markossian, T., Johnson, A., Dong, F., \& Bayakly, R. (2014). Geographic Residency Status and Census Tract Socioeconomic Status as Determinants of Colorectal Cancer Outcomes. American Journal of Public Health, 104(3), e63-e71. https://doi.org/10.2105/AJPH.2013.301572

Holsten, J. E. (2009). Obesity and the community food environment: a systematic review. Public Health Nutrition, 12(03), 397-405. https://doi.org/10.1017/S1368980008002267

Irigaray, P., Newby, J. A., Clapp, R., Hardell, L., Howard, V., Montagnier, L., ... Belpomme, D. (2007). Lifestyle-related factors and environmental agents causing cancer: An overview. Biomedicine \& Pharmacotherapy, 61(10), 640-658.

Jambon, C., Dejardin, O., Morlais, F., Pornet, C., Bouvier, V., \& Launoy, G. (2010). Déterminants socio-géographiques de la prise en charge des cancers en France - Exemple des cancers colorectaux incidents entre 1997 et 2004 dans le département du Calvados. Revue d'Épidémiologie et de Santé Publique, 58(3), 207-216. https://doi.org/10.1016/j.respe.2010.01.004

Jooste, V., Grosclaude, P., Remontet, L., Launoy, G., Baldi, I., Molinié, F., ... French Network of Cancer Registries (FRANCIM). (2013). Unbiased estimates of long-term net survival of solid cancers in France. International Journal of Cancer. Journal International Du Cancer, 132(10), 2370-2377. https://doi.org/10.1002/ijc.27857

Kangas, M., Bovbjerg, D. H., \& Montgomery, G. H. (2008). Cancer-related fatigue: a systematic and meta-analytic review of non-pharmacological therapies for cancer patients. Psychological Bulletin, 134(5), 700-741. https://doi.org/10.1037/a0012825

Kihal-Talantikite, W., Padilla, C. M., Lalloue, B., Rougier, C., Defrance, J., Zmirou-Navier, D., \& Deguen, S. (2013). An exploratory spatial analysis to assess the relationship between deprivation, noise and infant mortality: an ecological study. Environmental Health: A Global Access Science Source, 12, 109. https://doi.org/10.1186/1476-069X-12-109

Korda, R. J., Butler, J. R., Clements, M. S., \& Kunitz, S. J. (2007). Differential impacts of health care in Australia: trend analysis of socioeconomic inequalities in avoidable mortality. International Journal of Epidemiology, 36(1), 157-165. https://doi.org/10.1093/ije/dyl282

Künzli, N., Kaiser, R., Medina, S., Studnicka, M., Chanel, O., Filliger, P., ... Sommer, H. (2000). Public-health impact of outdoor and traffic-related air pollution: a European assessment. The Lancet, 356(9232), 795-801. https://doi.org/10.1016/S0140-6736(00)02653-2 
Le Corroller-Soriano, A.-G., Malavolti, L., \& Mermilliod, C. (2008). La vie deux ans après le diagnostic du cancer: une enquête en 2004 sur les conditions de vie des malades. (des relations sociales et de la solidarité $\mathrm{D}$. de la recherche France. Ministère du travail \& Institut national de la santé et de la recherche médicale, Éd.). Paris, France: La documentation française.

Leal, C., \& Chaix, B. (2011). The influence of geographic life environments on cardiometabolic risk factors: a systematic review, a methodological assessment and a research agenda. Obesity Reviews: An Official Journal of the International Association for the Study of Obesity, 12(3), 217-230. https:// doi.org/10.1111/j.1467-789X.2010.00726.x

Leclerc, A., Kaminski, M., \& Lang, T. (2008). Inégaux face à la santé: du constat à l'action (Vol. 1-1). Paris, France: la Découverte : Inserm.

Léonard, C., Stordeur, S., \& Roberfroid, D. (2009). Association between physician density and health care consumption: a systematic review of the evidence. Health Policy (Amsterdam, Netherlands), 91(2), 121-134. https://doi.org/10.1016/j.healthpol.2008.11.013

Lucas-Gabrielli V, Tonnelier F \& Vigneron E. Une typologie des paysages socio-sanitaires en France (1998). Paris, France: CREDES, 95 p.

Lurkin, A., Ducimetière, F., Vince, D. R., Decouvelaere, A.-V., Cellier, D., Gilly, F. N., ... RayCoquard, I. (2010). Epidemiological evaluation of concordance between initial diagnosis and central pathology review in a comprehensive and prospective series of sarcoma patients in the Rhone-Alpes region. BMC Cancer, 10, 150. https://doi.org/10.1186/1471-2407-10-150

Maass, R., Lindstrøm, B., \& Lillefjell, M. (2014). Exploring the relationship between perceptions of neighbourhood-resources, sense of coherence and health for different groups in a Norwegian neighbourhood. Journal of Public Health Research, 3(1). Consulté à l'adresse http://www.jphres.org/ index.php/jphres/article/view/208

Mackenbach, J. P. (2012). The persistence of health inequalities in modern welfare states: the explanation of a paradox. Social Science \& Medicine (1982), 75(4), 761-769. https://doi.org/10.1016/ j.socscimed.2012.02.031

Mastrangelo, G., Coindre, J.-M., Ducimetière, F., Dei Tos, A. P., Fadda, E., Blay, J.-Y., ... Rossi, C. R. (2012). Incidence of soft tissue sarcoma and beyond: A population-based prospective study in 3 European regions. Cancer. https://doi.org/10.1002/cncr.27555

Meijer, M., Röhl, J., Bloomfield, K., \& Grittner, U. (2012). Do neighborhoods affect individual mortality? A systematic review and meta-analysis of multilevel studies. Social Science \& Medicine, 74(8), 1204-1212. https://doi.org/10.1016/j.socscimed.2011.11.034

Onega, T., Duell, E. J., Shi, X., Wang, D., Demidenko, E., \& Goodman, D. (2008). Geographic access to cancer care in the U.S. Cancer, 112(4), 909-918. https://doi.org/10.1002/cncr.23229

Penel, N., Pasquier, D., \& Vanseymortier, L. (2007). Quelques éléments particuliers concernant l'épidémiologie des sarcomes. Oncologie, 9(2), 84-87. https://doi.org/10.1007/s10269-006-0578-9

Pornet, C., Delpierre, C., Dejardin, O., Grosclaude, P., Launay, L., Guittet, L., ... Launoy, G. (2012). Construction of an adaptable European transnational ecological deprivation index: the French version. Journal of Epidemiology and Community Health, 66(11), 982-989. https://doi.org/10.1136/ jech-2011-200311

Raymond, G., \& Pampalon, R. (2003). Indice de défavorisation matérielle et sociale : son application au secteur de la santé et du bien-être. Santé, Société et Solidarité, 2(1), 191-208. https:// doi.org/10.3406/oss.2003.932 
Rey, G., Jougla, E., Fouillet, A., \& Hémon, D. (2009). Ecological association between a deprivation index and mortality in France over the period 1997 -2001: variations with spatial scale, degree of urbanicity, age, gender and cause of death. BMC Public Health, 9, 33. https://doi.org/ 10.1186/1471-2458-9-33

Rican, S., Salem, G., Roudier-Daval, C., \& Weill, A. (2004). Géographie de l'incidence des cancers en France. Oncologie, 6(8), 540-547. https://doi.org/10.1007/s10269-005-0126-z

Riva, M., Gauvin, L., \& Barnett, T. A. (2007). Toward the next generation of research into small area effects on health: a synthesis of multilevel investigations published since July 1998. Journal of Epidemiology and Community Health, 61(10), 853-861. https://doi.org/10.1136/jech.2006.050740

Rose, G. (1985). Sick individuals and sick populations. International journal of epidemiology, 14(1), 32-38.

Rossi, C. R., Vecchiato, A., Mastrangelo, G., Montesco, M. C., Russano, F., Mocellin, S., ... RayCoquard, I. (2013). Adherence to treatment guidelines for primary sarcomas affects patient survival: a side study of the European CONnective TIssue CAncer NETwork (CONTICANET). Annals of Oncology: Official Journal of the European Society for Medical Oncology / ESMO, 24(6), 1685-1691. https://doi.org/10.1093/annonc/mdt031

Salem, G., Rican, S., Jougla, E., Suss, C., \& Berthod-Wurmser, M. (2000). Atlas de la santé en France. Volume 1, Les causes de décès. Montrouge : J. Libbey Eurotext.

Salem, G., Rican, S., Kürzinger, M.-L., Roudier-Daval, C., \& Khayat, D. (2006). Atlas de la santé en France. Volume 2, Comportements et maladies.

Saunders, L., Kadhel, P., Costet, N., Rouget, F., Monfort, C., Thomé, J.-P., ... Multigner, L. (2014). Hypertensive disorders of pregnancy and gestational diabetes mellitus among French Caribbean women chronically exposed to chlordecone. Environment International, 68, 171-176. https:// doi.org/10.1016/j.envint.2014.03.024

Smith, K. R., Brown, B. B., Yamada, I., Kowaleski-Jones, L., Zick, C. D., \& Fan, J. X. (2008).

Walkability and body mass index density, design, and new diversity measures. American Journal of Preventive Medicine, 35(3), 237-244. https://doi.org/10.1016/j.amepre.2008.05.028

Sogaard, M., Thomsen, R. W., Bossen, K. S., Sorensen, H. T., \& Norgaard, M. (2013). The impact of comorbidity on cancer survival: a review. Clinical Epidemiology, 5(Suppl 1), 3-29. https://doi.org/ 10.2147/CLEP.S47150

Tanke, M. A. C., \& Ikkersheim, D. E. (2012). A new approach to the tradeoff between quality and accessibility of health care. Health Policy, 105(2-3), 282-287. https://doi.org/10.1016/j.healthpol. 2012.02.016

Townsend P (1987). Deprivation. Journal of Social Policy, 16(2):125-146.

Trugeon, A., Thomas, N., Michelot, F., \& Fédération nationale des observatoires régionaux de santé (France). (2010). Inégalités socio-sanitaires en France : de la région au canton (Issy-lesMoulineaux : Masson).

Vallée, J., \& Chauvin, P. (2012). Investigating the effects of medical density on health-seeking behaviours using a multiscale approach to residential and activity spaces. Results from a prospective cohort study in the Paris metropolitan area, France. International Journal of Health Geographics, 11(1), 54. https://doi.org/10.1186/1476-072X-11-54

Van Hulst, A., Thomas, F., Barnett, T. A., Kestens, Y., Gauvin, L., Pannier, B., \& Chaix, B. (2012). A typology of neighborhoods and blood pressure in the RECORD Cohort Study. Journal of Hypertension, 30(7), 1336-1346. https://doi.org/10.1097/HJH.ob013e3283544863 
Weden, M. M., Bird, C. E., Escarce, J. J., \& Lurie, N. (2011). Neighborhood archetypes for population health research: Is there no place like home? Health \& Place, 17(1), 289-299. https:// doi.org/10.1016/j.healthplace.2010.11.002

\section{ANNEXES}

Figure 6 : Variables géographiques intégrées dans l'analyse multivariée des Iris en Rhône-Alpes

\begin{tabular}{|c|c|c|c|c|c|}
\hline Dimensions & Caractéristiques & Variables & Données et date & Echelle & Source \\
\hline \multirow{7}{*}{ PHYSIQUE } & \multirow{3}{*}{$\begin{array}{l}\text { Aménités et } \\
\text { nuisances }\end{array}$} & Qualité environnementale & \begin{tabular}{|c|} 
Part de la superficie occupée par des zones industrielles, réseaux \\
de communications, mines, chantiers et décharges (2006)
\end{tabular} & Commune & $\begin{array}{c}\text { Corine Land } \\
\text { Cover }\end{array}$ \\
\hline & & $\begin{array}{c}\text { Commerces et services de } \\
\text { proximité }\end{array}$ & $\begin{array}{c}\text { Nombre de types d'équipements parmi la gamme des } \\
\text { services de proximité (2007) }\end{array}$ & Iris & INSEE \\
\hline & & $\begin{array}{c}\text { Commerces et services } \\
\text { courants }\end{array}$ & Temps d'accès en minutes aux équipements intermédiaires (2006) & Bassin Vie & INSEE \\
\hline & \multirow{2}{*}{ Cadredevie } & Salubrité du logement & $\begin{array}{c}\text { Proportion des résidences principales équipées de } \\
\text { salle de bains (2006) }\end{array}$ & IRIS & INSEE \\
\hline & & Surpopulation du logement & Nombre de pièces par habitant (2006) & IRIS & INSEE \\
\hline & \multirow{2}{*}{$\begin{array}{c}\text { Politiques } \\
\text { d'aménagement }\end{array}$} & Dynamique foncière & Part des logements construits entre 1999 et 2009 & Commune & Datar \\
\hline & & $\begin{array}{c}\text { Organisation des } \\
\text { transports }\end{array}$ & $\begin{array}{l}\text { Part des déplacements domicile-travail } \\
\text { en transports en commun (2007) }\end{array}$ & Aire Urbaine & Certu \\
\hline \multirow{5}{*}{ SOCIALE } & \multirow{3}{*}{$\begin{array}{l}\text { Profil socio- } \\
\text { économique }\end{array}$} & $\begin{array}{l}\text { Catégorie socio- } \\
\text { professionnelle }\end{array}$ & Proportion ouvriers et employés chez 15 ans et plus (2009) & IRIS & INSEE \\
\hline & & Revenu & Revenu médian par Unité de Consommation (2006) & IRIS & INSEE \\
\hline & & Dynamisme économique & Taux de chômage (2007) & Commune & INSEE \\
\hline & Capital social & Personnes isolées & $\begin{array}{c}\text { Proportion de personnes vivant seules ou dans une famille } \\
\text { monoparentale (2007) }\end{array}$ & Commune & INSEE \\
\hline & $\begin{array}{c}\text { Comportements } \\
\text { desanté }\end{array}$ & Dépistage du cancer & Taux participation dépistage cancer du sein (2005-2006) & Commune & Associations \\
\hline \multirow{3}{*}{ MEDICALE } & \multirow{3}{*}{$\begin{array}{l}\text { Démographie } \\
\text { libérale }\end{array}$} & Densité généralistes & $\begin{array}{c}\text { Nombre d'équivalents temps plein de médecins généralistes } \\
\text { pour } 100000 \text { habitants en } 2010\end{array}$ & Commune & IRDES \\
\hline & & Densité spécialistes & $\begin{array}{l}\text { Densité de médecins spécialistes au 31/12/2006 } \\
\text { (Nombre de spécialistes/population*100000) }\end{array}$ & Bassin Vie & CNAM \\
\hline & & Densité Para-Médicaux & $\begin{array}{l}\text { Nombre de personnels para-médicaux } \\
\text { pour } 100000 \text { habitants (2010) }\end{array}$ & Cantons & INSEE \\
\hline
\end{tabular}


Figure 7 : Tableau croisée des moyennes pour chaque variable géographique de l'analyse multivariée en fonction du type de territoire

\begin{tabular}{|c|c|c|c|c|c|c|c|}
\hline Variables & $\begin{array}{c}\text { Quartiers } \\
\text { métroplitains }\end{array}$ & $\begin{array}{l}\text { Quartiers } \\
\text { populaires }\end{array}$ & $\begin{array}{l}\text { Pôles } \\
\text { urbains }\end{array}$ & $\begin{array}{c}\text { Zones } \\
\text { résidentielles }\end{array}$ & $\begin{array}{c}\text { Espaces } \\
\text { périurbains }\end{array}$ & $\begin{array}{l}\text { Espaces } \\
\text { ruraux }\end{array}$ & Rhône-Alpes \\
\hline $\begin{array}{l}\text { Part de la superficie occupée par des } \\
\text { nuisances (\%) }\end{array}$ & 6,7 & 21,9 & 9,9 & 4,3 & 1,5 & 0,7 & 4,9 \\
\hline $\begin{array}{l}\text { Nombre de types d'équipements de } \\
\text { proximité }\end{array}$ & 16,0 & 15,0 & 18,0 & 15,0 & 13,0 & 7,0 & 12,0 \\
\hline $\begin{array}{c}\text { Accès aux équipements } \\
\text { intermédiaires (en minutes) }\end{array}$ & 9,9 & 11,8 & 17,7 & 14,1 & 17,8 & 23,0 & 18,2 \\
\hline Proportion de salle de bains (\%) & 96,1 & 95,9 & 97,5 & 98,0 & 97,5 & 94,8 & 96,3 \\
\hline Nombre de pièces par habitant & 1,6 & 1,5 & 1,7 & 1,8 & 1,8 & 1,9 & 1,8 \\
\hline $\begin{array}{l}\text { Part des logements construits } \\
\text { depuis } 1999(\%)\end{array}$ & 2,6 & 6,7 & 9,5 & 13,2 & 15,6 & 8,4 & 10,5 \\
\hline $\begin{array}{l}\text { Déplacements domicile-travail en } \\
\text { transports en commun (\%) }\end{array}$ & 18,0 & 15,0 & 8,7 & 13,1 & 8,7 & 5,1 & 9,0 \\
\hline Part des ouvriers-employés (\%) & 19,7 & 33,3 & 31,8 & 23,9 & 31,8 & 30,2 & 30,0 \\
\hline $\begin{array}{l}\text { Revenu Médian par Unité de } \\
\text { Consommation }(\epsilon)\end{array}$ & 21397 & 14666 & 16393 & 22192 & 18127 & 15499 & 17206 \\
\hline Taux de chômage (\%) & 7,5 & 9,4 & 8,1 & 4,4 & 4,9 & 6,1 & 6,2 \\
\hline Part des personnes vivant seules (\%) & 32,7 & 30,6 & 27,8 & 16,7 & 15,8 & 19,9 & 21,1 \\
\hline $\begin{array}{l}\text { Taux de participation au dépistage du } \\
\text { cancer du sein (\%) }\end{array}$ & 47,3 & 46,1 & 42,0 & 52,1 & 46,3 & 42,5 & 45,0 \\
\hline $\begin{array}{l}\text { Nombre d'ETP* de médecins } \\
\text { généralistes pour } 100000 \text { habitants }\end{array}$ & 72,9 & 77,1 & 89,7 & 59,2 & 52,6 & 35,8 & 54,9 \\
\hline $\begin{array}{c}\text { Nombre de médecins spécialistes } \\
\text { pour } 100000 \text { habitants }\end{array}$ & 126,6 & 118,8 & 97,9 & 107,5 & 50,6 & 27,9 & 64,7 \\
\hline $\begin{array}{c}\text { Nombre de personnels paramédicaux } \\
\text { pour } 100000 \text { habitants }\end{array}$ & 298,5 & 271,5 & 394,7 & 332,2 & 280,0 & 249,6 & 286,4 \\
\hline Nombre d'ris & 193 & 449 & 410 & 417 & 1091 & 1417 & 3977 \\
\hline Population moyenne par Iris & 2602 & 2136 & 2542 & 2529 & 1431 & 603 & 1505 \\
\hline Population totale & 502109 & 1042067 & 1135376 & 890706 & 1561659 & 854749 & 5986666 \\
\hline $\begin{array}{l}\text { Part de la population en } \\
\text { Rhône-Alpes (en\%) }\end{array}$ & 8,4 & 17,4 & 19,0 & 14,9 & 26,1 & 14,3 & 100 \\
\hline
\end{tabular}

\section{RÉSUMÉS}

Agir efficacement contre les inégalités géographiques face aux cancers implique une meilleure compréhension du processus aboutissant à ces inégalités. Une méthodologie interdisciplinaire, traitant conjointement les multiples dimensions par lesquelles le territoire impacte la santé et retraçant l'évolution pronostique des patients, permettrait de répondre à ce besoin. La cohorte EMS, retraçant la prise en charge de patients atteints de sarcomes (cancers rares) en RhôneAlpes, a été analysée suivant ces principes méthodologiques. L'analyse de 15 variables géographiques ayant une influence sur la santé a permis de distinguer six types de territoires rhônalpins. Cette typologie a ensuite été croisée avec les données de la cohorte EMS pour étudier les pertes de chances propres à chaque type de territoire. La surmortalité des pôles urbains est liée à l'incidence plus importante des sarcomes, quand celle des quartiers populaires et des espaces ruraux s'expliquent davantage par des pertes de chances de survie au cours de la prise en charge.

Cancers inequalities in France are among the highest in developed countries and these gaps seem to be growing in the last decades. Territorial inequalities of cancers are analyzed by mapping, which showed higher mortality rates in the North-East Regions of France. At the local scale, standardized mortality rates are two times higher in some areas in the North as other areas in the South-East. Epidemiological studies, mostly based on multilevel analysis, evidence the impact of physical environment, deprivation or health care access on health outcomes. But to identify contextual effects are not sufficient to understand how cancer inequalities are built and how patient's life context contributes to this process. As epidemiological approach is splitting contextual effects according to health outcomes, geographical approach may have to explain 
how these contextual effects lead to cancer inequalities, by using territorial typology to summarize these contextual effects. Comparing health outcomes according to this territorial classification may help to understand territory's ability to generate health inequalities.

Several stages across the cancer continuum are implied in the building of the cancer inequalities. This medical process has to be reconstructed to determine whether mortality inequalities are generated by a higher incidence or a lower survival. Moreover, lower survival may be linked to the worse prognosis of patients at diagnosis or to the lower quality of management according to cancer care facilities. Evolution of patients' prognosis may be reconstructed, thanks to clinical data, in order to identify the most influent steps during this medical process.

As a result, to understand the way geographical inequalities of cancers are building requires a multidisciplinary methodology, considering the territory's contribution as a whole and using longitudinal clinical data. But to reconstitute this medical process is quite difficult actually because few longitudinal and exhaustive data are available. The EMS cohort represents an opportunity to test and discuss this methodological approach. This cohort includes all sarcoma (rare cancer) patients diagnosed in 2005 and 2006, in the Rhône-Alpes Region, and collects clinical data from the diagnosis to the patient follow-up. For this geographical analysis of the EMS cohort, we used a territorial typology generated thanks to multivariate analysis of 15 geographical variables known for their impact on health. Strong differences are noticed in terms of environment exposures, deprivation and health care access between the six types of territory (metropolitan neighborhoods, working-class neighborhoods, urban districts, residential areas, periurban areas, rural areas). This typology seems to be relevant to study geographical inequalities because it enables to distinguish populations which are not exposed to the same risks through their life context. A logistic regression including stage, age and histological subtype, as clinical factors influencing prognosis, estimates the patients' prognosis at diagnosis. This prognosis score seems to be quite predictive because only 7\% of "good prognosis" patients will be dead five years later, whereas this five years death rate raises to $80 \%$ for the worse prognosis patients.

Analysis of geographical inequalities for sarcoma patients in the Rhône-Alpes Region shows the diversity of situations leading to inequalities of mortality. Indeed, the higher mortality in three types of territories has to be attributed to three different processes. In the case of the urban hub, this high mortality is linked to the higher incidence of sarcoma, as survival rate for patients of these districts is very close to the regional average. As incidence and prognosis risk in the working-class neighborhoods are quite similar to the regional average, higher mortality is due to the loss of survival odds in the course of treatments, probably because of a less optimal management. Despite the second lower incidence among the six types of territories, the worse prognosis of patients (more late-stage diagnosis) and the loss of survival odds during cancer management explained the high mortality rated in the rural areas.

Thanks to the EMS cohort's analysis, we assess the potential of a multidisciplinary methodology studying the territory's ability to generate geographical inequalities of cancers. Territorial typology, produced without health outcomes data, may be used for every health studies as a synthetic index of the territory and also evidence strong inequalities of health according to people's life contexts. As public policies struggle to be successful on this issue, to identify key events in the medical process leading to cancer inequalities may improve the territorialization and the efficiency of these policies. Territories with high risk before diagnosis would be focused on prevention and early diagnosis, whereas those more disadvantage during the management would lean towards cancer care quality, access to hospitals and cancer expertise and patient compliance. 
INDEX

Mots-clés : cancer, inégalités géographiques, cohorte EMS

Index géographique : Rhônes-Alpes

\section{AUTEURS}

\section{YOHAN FAYET}

Chercheur assistant au Centre Léon Bérard, EA 7425 HESPER (Health Services and Performance Research)

\section{VIRGINIE CHASLES}

Maitre de Conférences en Géographie, Université Jean Moulin Lyon 3, EA 7425 HESPER

\section{FRANÇOISE DUCIMETIÈRE}

Chef de projets, Centre Léon Bérard Lyon

\section{PR ISABELLE RAY-COQUARD}

Oncologue médical PU-PH, Centre Léon Bérard Lyon, EA 7425 HESPER 